\title{
COVID-19, a disease of the nicotinic cholinergic system? Nicotine may be protective
}

\author{
Konstantinos Farsalinos ${ }^{1}$, Raymond Niaura ${ }^{2}$, Konstantinos Poulas ${ }^{3}$ \\ 1 University of West Attica \\ 2 New York University \\ 3 University of Patras
}

\begin{abstract}
Corona Virus Disease 2019 (COVID-19) is a disease caused by a novel coronavirus, SARS-CoV-2. On 23 March, we presented for the first time evidence of a low prevalence of smoking among hospitalized patients with COVID-19 in China. On 3 April, we were the first to establish the hypothesis that nicotine may be beneficial for COVID19 patients and should be evaluated in clinical trials due to its anti-inflammatory properties. We present briefly the hypothesis that COVID-19 could be a disease of the nicotinic cholinergic system.

Additionally we emphasize that researchers should be cautious when presenting previously-published (or pre-published) work as their own "novel" work. Copying methodologies and reporting previously-presented hypotheses without citing the original authors, especially when publication and pre-publication dates are documented, can be considered plagiarism and a breach of intellectual property copyright.
\end{abstract}

Corona Virus Disease 2019 (COVID-19) is a disease caused by a novel coronavirus, SARSCoV-2. On 23 March, we presented for the first time evidence of a low prevalence of smoking among hospitalized patients with COVID-19 in China. On 3 April, we were the first to establish the hypothesis that nicotine may be beneficial for COVID-19 patients and should be evaluated in clinical trials due to its anti-inflammatory properties.

While in many cases the disease is mild, severe COVID-19 involves a hyper-inflammatory response, commonly called cytokine storm. This is characterized by the release of proinflammatory cytokines that can lead to Acute Respiratory Distress Syndrome and death. The cholinergic anti-inflammatory pathway is mediated by nAChRs and can control 
inflammation. it functions as an immunomodulator through bi-directional communication between the immune and nervous systems.

The clinical manifestations of cytokine storm observed in COVID-19 patients could be linked to a dysfunction of the cholinergic anti-inflammatory pathway. At the same time, several patients experience neurological symptoms that could be explained by the invasion of the virus to the terminal area of afferent vagus fibers or the origin of the efferent vagus fibers, and further dysregulation of the inflammatory response. Anosmia has been experienced by several COVID-19 patients. Interestingly, anosmia is a premature clinical manifestation of Parkinson's disease. Up to 95\% of patients with Parkinson's disease experience anosmia; this may appear years before motor symptoms. Smoking reduces the risk for anosmia in Parkinson's disease patients. The cause of olfactory dysfunction in these patients is impaired cholinergic transmission, and the same mechanism may be implicated in COVID-19-induced anosmia. . Thromboembolic complications, activation of platelets and endothelial damage with increased vascular permeability also indicate ineffective control by the nicotinic cholinergic system. Considering that most of the manifestations of COVID-19 can be manifestations of impaired nAChRs function, we make the hypothesis that COVID-19 could be a disease of the nicotinic cholinergic system. We propose that nicotine could be used therapeutically and should be urgently evaluated in clinical trials. More details will be presented in an upcoming publication.

We emphasize that researchers should be cautious when presenting previously-published (or pre-published) work as their own "novel" work. Copying methodologies and reporting previously-presented hypotheses without citing the original authors, especially when publication and pre-publication dates are documented, can be considered plagiarism and a breach of intellectual property copyright. 